\title{
The serotonin transporter gene polymorphism (5-HTTLPR) and irritable bowel syndrome: a meta-analysis of 25 studies
}

\author{
Zhi-Feng Zhang ${ }^{1}$, Zhi-Jun Duan ${ }^{1 *}$, Li-Xia Wang ${ }^{1}$, Dong Yang ${ }^{1}$, Gang Zhao ${ }^{1}$ and Lin Zhang ${ }^{2}$
}

\begin{abstract}
Background: The results of previous studies assessing the association between the 5-HTTLPR polymorphism of serotonin transporter gene and irritable bowel syndrome (IBS) are inconsistent. The aim of this study was to clarify the association between the 5-HTTLPR mutation and the presence of IBS and its subtypes with a meta-analysis of 25 studies.
\end{abstract}

Methods: A thorough search for case-control studies evaluating the association between the 5-HTTLPR polymorphism of serotonin transporter gene and the presence of IBS was carried out in four electronic databases. A meta-analysis was performed in accordance with the Cochrane Handbook for systemic reviews.

Results: A total of 25 articles with 3443 IBS cases and 3359 controls were included into our meta-analysis. No significant association was found between this polymorphism and IBS in all populations. Whereas the LL genotype was demonstrated to be a risk factor for constipation predominant IBS (IBS-C) development in the overall population (LL vS SS: $O R=1.570,95 \% \mathrm{Cl}=1.147-2.148, \mathrm{P}=0.005$, $\mathrm{Bon}=0.030 ; \mathrm{LL}$ vS $L \mathrm{~S}: \mathrm{OR}=1.658,95 \% \mathrm{Cl}=1.180-2.331, \mathrm{P}=0.004$, Bon $=0.024 ; \mathrm{LL}$ vs $L S / S S: O R=1.545,95 \% \mathrm{Cl}=1.187-2.012, \mathrm{P}=0.001$, $\mathrm{Bon}=0.006$ ). In the analysis of different ethnicities, $\mathrm{L}$ allele and $\mathrm{LL}$ genotype were significantly associated with increased IBS-C risk in the East Asian population ( $\mathrm{L}$ vs S: $\mathrm{OR}=1.487,95 \% \mathrm{Cl}=1.139-1.941, \mathrm{P}=0.003$, Bon $=0.018 ; \mathrm{LL}$ vs SS: $\mathrm{OR}=2.575,95 \% \mathrm{Cl}=1.741-3.808, \mathrm{P}=0.000$, Bon = 0.000; LL vS LS: $O R=3.084,95 \% \mathrm{Cl}=2.017-4.715, \mathrm{P}=0.000$, $\mathrm{Bon}=0.000 ; \mathrm{LL}$ vS $\mathrm{LS} / \mathrm{SS}: \mathrm{OR}=2.759,95 \% \mathrm{Cl}=1.933-3.938$, $P=0.000$, Bon $=0.000$ ), but not in the Caucasian population.

Conclusions: Different from the conclusions of the earlier meta-analyses, the 5-HTTLPR mutation affects IBS-C but not IBS-D and IBS-M development and this effect only exists in the East Asian population but not other populations.

\section{Background}

Irritable bowel syndrome (IBS) mainly affecting lower gastrointestinal (GI) tracts is a chronic functional GI disorder with an obvious heterogeneity among affected patients. The characteristic presentation of IBS is recurrent abdominal pain accompanied with altered bowel habits $[1,2]$. According to the Rome III criteria, IBS is categorized into diarrhea predominant IBS (IBS-D), constipation predominant IBS (IBS-C), mixture of diarrhea and constipation IBS (IBS-M) and un-subtyped IBS [3]. IBS is a common disorder encountered by gastroenterologists and its prevalence is approximately $10 \%$ [4]. IBS can impair the

\footnotetext{
* Correspondence: cathydoctor@yahoo.com

'Department of Gastroenterology, The First Affiliated Hospital of Dalian

Medical University, 116000 Dalian, Liaoning province, China

Full list of author information is available at the end of the article
}

quality of life and the work efficiency of affected patients. Due to the elusive etiology of IBS, there is still no curative therapy for this condition. Therapy for relieving symptoms is still the mainstay for IBS patients. Long term symptomatic treatment results in an economic burden on not only patients and their families but also healthcare systems [5]. Despite lacking of the definite etiology, some advancements have been achieved in understanding the pathophysiology underlying IBS development in recent years. Gut motility dysfunction, visceral hypersensitivity and psychopathological factors have been implicated to play key roles in the development of IBS $[1,3]$.

Serotonin is a neurotransmitter existing in both central nervous system (CNS) and GI tracts, and regulates GI tracts motility, visceral sensation and mucosal secretion through a paracrine signaling pathway [6]. Previous studies 
have shown that elevated plasma serotonin is associated with IBS-D and decreased plasma serotonin is associated with IBS-C [7]. Once serotonin is secreted from enterochromaffin (EC) cells, serotonin reuptake transporter (SERT) will be activated to reuptake serotonin back into EC cells and attenuate the effect of serotonin in GI tracts subsequently [8]. Balance between these two opposite processes determining the net tone of serotonin in GI tracts is critical to the maintenance of normal gut functions, especially of GI tracts motility. Changes of the SERT activity would break this balance and could be involved in the development of IBS theoretically. A polymorphism of SERT gene (5-HTTLPR) with a short (S) variation of 14 repeats and a long $(\mathrm{L})$ variation of 16 repeats has been proven to influence the activity of SERT [9-11]. So 5-HTTLPR is very likely to be associated with the development of IBS. Casecontrol studies about 5-HTTLPR were conducted to verify this hypothesis. Some studies demonstrated a positive association between this polymorphism and IBS $[10,11]$, while another study failed to confirm this association [12]. A meta-analysis of 8 studies conducted in 2007 tried to reach a definite conclusion and showed a negative result [13]. However, the small sample size of this meta-analysis weakened its strength of evidence and this meta-analysis did not terminate the controversy about 5-HTTLPR in IBS development. Another meta-analysis published in a letter showed a positive association between this polymorphism and IBS-C [14]. However, this meta-analysis did not include all the published articles and did not assess the association in different ethnic groups. The third one included most of the publish articles and concluded that 5-HTTLPR was associated with IBS [15]. The third metaanalysis also had limitations and did not assess the association in different IBS subtypes. The association may be different in different IBS subtypes and different ethnic groups. Hence we performed this meta-analysis including all published studies accompanied with ethnic subgroup analyses and IBS subtype analyses to clarify whether 5HTTLPR was associated with the development of IBS and its subtypes.

\section{Methods}

\section{Searching strategies}

Case-control studies evaluating the association between the 5-HTTLPR polymorphism and IBS were searched in PubMed, Embase and Web of Science with the combinations of the following searching terms: "irritable bowel syndrome", "IBS", "serotonin", "5-Hydroxytryptamine", "5HT", "polymorphism", "polymorphisms", "single nucleotide", "allele" and "genotype". We also searched China National Knowledge Infrastructure (CNKI) for additional relevant researches. Reference lists of each article, relevant meta-analyses and reviews were searched as well. The last searching date was July 12, 2013.

\section{Study selection criteria}

Studies included in this meta-analysis fulfilled the following selection criteria: 1) Case-control studies with a healthy control arm. 2) Studies evaluating the association between the 5-HTTLPR polymorphism and IBS. 3) IBS diagnosis according to Rome I or II or III criteria. 4) Articles providing allele and genotype frequencies or odds ratios (OR) and $95 \%$ confidence intervals (95\% CI). Exclusion criteria were as the follows: 1) Republication. 2) Family based studies. If the data of a study was published in different articles, the article with the largest sample size would be included in this meta-analysis. If the data of a study was published not only as an abstract but also as a full text article in different magazines, we selected the full text article to analyze in order to assess the quality of the research more comprehensively. During study selection, two authors would read the highly relevant articles independently to determine eligible studies. A third author would be consulted and the decision would be reached through discussions when a disagreement was encountered.

\section{Data abstraction}

A standard data extraction form was used to abstract data by two investigators. And the data extraction items included: first author, publication year, region where the study was conducted, ethnicity, case and control definition, allele and genotype frequencies in each group and the method of polymorphism detection. When we encountered an article neither in English nor in Chinese, would a professional translator be consulted to interpret the article.

\section{Assessment of the risks of bias}

Risks of bias were assessed with the following items: 1) Selection bias (cases and controls selections; selections based on disease subtypes), 2) Information bias (genotyping quality control procedures, genotyping under blind conditions and phenotype misclassification rate), 3) Confounding factors (ethnic origin between cases and controls, age and gender distribution between cases and controls).

\section{Quantitative data synthesis}

Hardy-Weinberg equilibrium in the control group of each study was assessed using the chi-square test, while $P<$ 0.05 was considered disequilibrium. Paired combinations of genotypes were used to determine the hereditary models: 1 ) an allelic analysis (L versus $\mathrm{S}$ ); 2) a genotypic analysis (LL versus SS, LL versus LS, LS versus SS) and 3) another genotypic analysis evaluating dominant or recessive effects of allele L (LL versus LS/SS, LL/LS versus SS). OR and its 95\% CI were calculated with the methods recommended by the Cochrane Collaboration [14]. Statistical 
heterogeneity among studies was detected with the Q test, with a value of $P<0.10$ indicating heterogeneity existence. The $I^{2}$ statistics was also employed to assess the risks of heterogeneity: $0 \%-40 \%$ meant no risk of heterogeneity, $30 \%-60 \%$ meant a low risk of heterogeneity, 50\%-90\% meant substantial heterogeneity and 75\%-100\% meant considerable heterogeneity [16]. The $I^{2}$ statistics less than $40 \%$ was used as the threshold to determine heterogeneity existence in this meta-analysis. If the $\mathrm{Q}$ test and the $I^{2}$ statistics both indicated no existence of heterogeneity, a fixed model with the Mantel-Haenszel method would be employed to pool data. Otherwise, a random model with the DerSimonian-Laird method would be applied to synthesize data. Funnel plots and Egger's test were used to examine publication bias $[17,18]$. The step down Bonferroni method was used for the multiple comparison adjustments [19]. Moreover, Student's t test and box plots were used to determine allele $\mathrm{L}$ variations among different ethnicities. Stata 11.0 software (StataCorp LP, College Station, Texas, USA) was used for meta-analysis, Hardy-Weinberg equilibrium (HWE) tests, Egger's test, Student's t test and box plots drawing. R 2.15.0 software (The R Foundation for Statistical Computing, http://cran.rstudio.com/) was used for step down Bonferroni adjustments (Bon). Values of $P<0.05$ were considered statistically significant for meta-analyses, Bonferroni adjustments, Egger's test and Student's t test.

\section{Results and discussion Results Characteristics of selected studies}

A total of 372 potentially relevant publications were identified from the four databases (PubMed: 73; Web of Science: 124; Embase: 165; CNKI: 10). After excluding studies not fulfilling our inclusion criteria including two abstracts not providing genotype frequencies, we included 25 articles ( 3 abstracts and 22 full text articles) with 3443 IBS cases and 3359 controls into our metaanalysis [10-12,20-41]. Among the selected studies, 11 studies were conducted in the Caucasian population, 9 studies were conducted in the East Asian population, two studies was conducted in the Indian population, one study was conducted in the Iranian population, one study was conducted in the Turkish population and one study was conducted in the Mexican population. In searching reference lists, relevant meta-analyses and reviews, no additional articles were identified. The flow chart of study selection is presented in Figure 1. And the characteristics of selected studies are illustrated in Table 1. The PRISMA Statement is illustrated in Additional file 1. The searching processes of Pubmed, Embase and Web of Science are illustrated in Additional file 2 .

\section{Assessment of the risks of bias}

Selection bias IBS cases were diagnosed and selected in accordance with the Rome criteria. Because of the evolution of the Rome criteria, all of the three Rome criteria were used by studies included in our meta-analysis. Two studies used Rome I criteria to diagnose IBS $[10,20]$, thirteen studies used Rome II criteria to diagnose IBS [11,21-31,33], nine studies used Rome III criteria to diagnose IBS [32,34-41] and one study selected patients from an IBS database [14]. Only four studies provided detailed descriptions of matching methods $[22,23,26,28]$. Measures were used to exclude IBS in controls in only seven studies [12,22,26,27,31,35,38]. Most studies selected IBS patients based on IBS subtypes [10-12,20-31,34-41].

Information bias Twenty four studies used allele-specific polymerase chain reaction (AS-PCR) to determine genotypes [10-12,20-31,33-41]. One study used polymerase chain reaction-restriction fragment length polymorphism (PCR-RFLP) to detect genotypes [32]. Two researches were carried out under blind conditions [30,39]. Four studies used the DNA sequencing method to confirm genotypes $[11,12,34,41]$. All experiments were repeated to ensure consistency for quality control purposes in one study [25]. No phenotype misclassification was reported in the selected studies.

Confounding factors Age and gender distributions were comparable among arms in nine studies [11,22,23,26,28, $31,34,35,37]$, while the other studies did not describe the age and gender distributions among groups. All studies matched cases and controls ethnically.

\section{Meta-analysis of the association between 5-HTTLPR polymorphism and IBS}

Detailed meta-analysis results, models used in each comparison and $P$ values for Bonferroni adjustment are shown in Table 2 and Table 3. A wide variation of allele $\mathrm{L}$ frequency between the East Asian population and the Caucasian population was found. L allele frequency of the East Asian population controls (29.627\%, 95\% CI = $19.532 \%-39.721 \%)$ was significantly lower than that of the Caucasian population controls $(52.182 \%, 95 \% \mathrm{CI}=$ 45.166\%-59.198\%) $(P=0.0003)$. The box plots are illustrated in Figure 2.

\section{Meta-analysis about IBS}

No significant association was found between this polymorphism and IBS in the overall population. In the analysis of the Caucasian population, only the LL vs LS contrast showed a positive result $(\mathrm{OR}=1.288,95 \% \mathrm{CI}=1.079-1.536$, $P=0.005$, Bon $=0.030$ ). There was no association between the 5-HTTLPR polymorphism and IBS in the East Asian, Iranian, Turkish, Indian and Mexican population. 


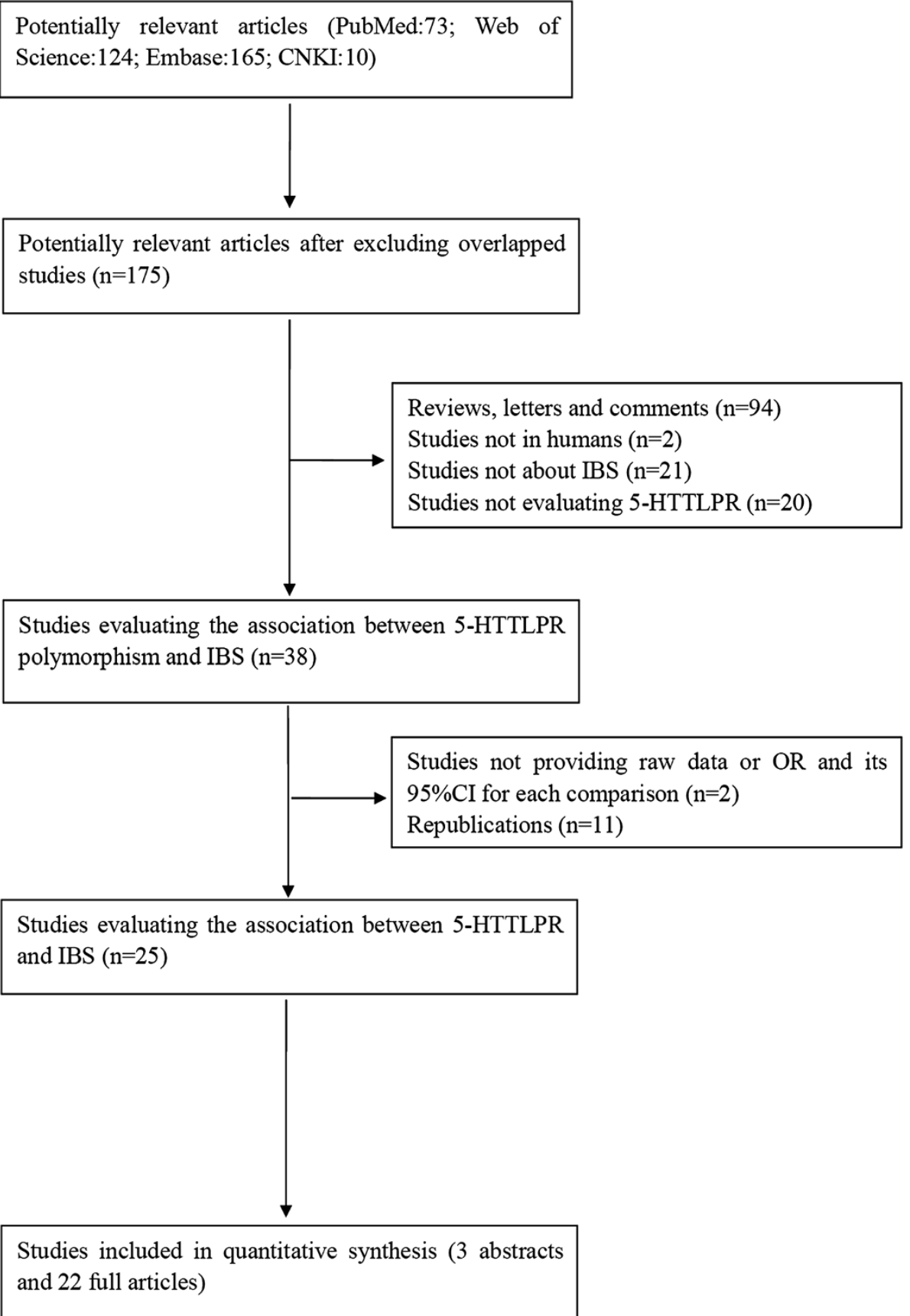

Figure 1 Flow diagram of the study selection process.

\section{Meta-analysis about IBS-C}

The LL genotype was a risk factor for IBS-C development in the overall population (LL vs SS: OR $=1.570$, 95\% CI $=1.147-2.148, P=0.005$, Bon $=0.030$; LL vs LS: $\mathrm{OR}=1.658,95 \% \mathrm{CI}=1.180-2.331, P=0.004$, Bon $=0.024$; $\mathrm{LL}$ vs $\mathrm{LS} / \mathrm{SS}$ : $\mathrm{OR}=1.545,95 \% \mathrm{CI}=1.187-2.012, P=0.001$, Bon $=0.006$ ). In the subgroup analysis of the East Asian population, L allele and LL genotype were significantly associated with increased IBS-C risk in a recessive way
$(\mathrm{L}$ vs $\mathrm{S}: \mathrm{OR}=1.487,95 \% \mathrm{CI}=1.139-1.941, P=0.003$, Bon $=$ 0.018 ; $\mathrm{LL}$ vs $\mathrm{SS}$ : $\mathrm{OR}=2.575,95 \% \mathrm{CI}=1.741-3.808, P=$ 0.000 , Bon $=0.000$; LL vs LS: OR $=3.084,95 \% \mathrm{CI}=2.017$ 4.715, $P=0.000$, Bon $=0.000 ; \mathrm{LL}$ vs LS/SS: OR $=2.759,95 \%$ $\mathrm{CI}=1.933-3.938, P=0.000$, Bon $=0.000$ ). Forest plots with positive results of the East Asian population are shown in Figure 3. However, there was no significant association between this polymorphism and IBS-C development in the Caucasian, Iranian, Turkish and Indian population. The 
Table 1 Characteristics of included studies evaluating the association between the 5-HTTLPR polymorphism and the presence of IBS

\begin{tabular}{|c|c|c|c|c|c|c|}
\hline Author & Year & Location & Ethnicity & Case and control selection & $\begin{array}{l}\text { SNP } \\
\text { method }\end{array}$ & $\begin{array}{l}\text { HWE } \\
\text { test }\end{array}$ \\
\hline \multirow[t]{2}{*}{ Pata C [20] } & 2002 & Turkey & Turkish & Case: patients diagnosed with Rome I criteria & AS-PCR & \\
\hline & & & & $\begin{array}{l}\text { Control: healthy volunteers without detailed descriptions } \\
\text { of matching methods }\end{array}$ & & $P=0.0196$ \\
\hline \multirow[t]{2}{*}{ Lee DY [21] } & 2004 & Korea & East Asian & Case: patients diagnosed with Rome II criteria & AS-PCR & \\
\hline & & & & $\begin{array}{l}\text { Control: healthy controls without detailed descriptions of } \\
\text { matching methods }\end{array}$ & & $P=0.8526$ \\
\hline Kim HJ [12] & 2004 & USA & Mostly Caucasian & $\begin{array}{l}\text { Case: patients selected from a database of } 752 \text { patients } \\
\text { with IBS }\end{array}$ & AS-PCR & \\
\hline
\end{tabular}

Wang BM [22]

2004 China

East Asian

Yeo A [10]

2004 North America Caucasian

Zhang XM [23]

2006 China

East Asian

Park JM [11]

2006 Korea

Whitehead WE [24] 2006 USA

Li Y [25]

2007 China

Saito YA [26]

Camilleri M [27]

2008 USA

Kohen R [28]

2009 USA

Schmulson M [29]

2009 Mexico

Niesler B [30]

2010 UK

Sikander A [31]

2009 India

Markoutsaki T [32] 2011 Greek

Jiang ZD [33]

2012 USA

East Asian

East Asian

Mexican

Caucasian

Indian

Caucasian
Control: healthy controls without detailed descriptions of matching methods

Case: patients diagnosed with Rome II criteria

Control: healthy contrtols matched for age and gender

Case: patients diagnosed with Rome I criteria

Control: healthy controls without detailed descriptions of matching methods

Case: patients diagnosed with Rome II criteria

Control: healthy controls matched for age and gender

Case: patients diagnosed with Rome II criteria

Control: healthy controls without detailed descriptions of matching methods

Mostly Caucasian Case: patients diagnosed with Rome II criteria

Control: healthy controls without detailed descriptions of matching methods

Case: patients diagnosed with Rome II criteria

Control: healthy controls without detailed descriptions of matching methods

Mostly Caucasia Case: patients diagnosed with Rome II criteria

Control: no IBS patients matched for age, race and gender

Mostly Caucasian Case: patients diagnosed with Rome II criteria

Control: healthy controls without detailed descriptions of matching methods

Mostly Caucasia Case: patients diagnosed with Rome II criteria

Control: healthy volunteers matched for age, race and gender

Case: patients diagnosed with Rome II criteria

Control: healthy controls without detailed descriptions of matching methods

Case: patients diagnosed with Rome II criteria

Control: healthy volunteers without detailed descriptions of matching methods

Case: patients diagnosed with Rome II criteria

Control: healthy controls without detailed description of matching methods

Case: patients diagnosed with Rome III criteria

Control: healthy volunteers without detailed description of matching methods

Mostly Caucasian Case: patients diagnosed with Rome II criteria

Control: healthy controls without detailed descriptions of matching methods
$P=0.9564$

AS-PCR

$P=0.1693$

AS-PCR

$$
P=0.7053
$$

AS-PCR

$P=0.9536$

AS-PCR

$P=0.1976$

AS-PCR

$P=0.2376$

AS-PCR

$P=0.5862$

AS-PCR

$P=0.2372$

AS-PCR

NC

AS-PCR

$P=0.1479$

AS-PCR

$P=0.0000$

AS-PCR

$P=0.5492$

AS-PCR

$P=0.4341$

PCR-RFLP

$P=0.6973$

AS-PCR

$P=0.1063$ 


\begin{tabular}{|c|c|c|c|c|c|c|}
\hline \multirow[t]{2}{*}{ Wang YM [34] } & 2012 & China & East Asian & Case: patients diagnosed with Rome III criteria & AS-PCR & \\
\hline & & & & $\begin{array}{l}\text { Control: healthy controls without detailed description of } \\
\text { matching methods }\end{array}$ & & $P=0.0000$ \\
\hline \multirow[t]{2}{*}{ Park CS [35] } & 2012 & Korea & East Asian & Case: patients diagnosed with Rome III criteria & AS-PCR & \\
\hline & & & & $\begin{array}{l}\text { Control: healthy controls without detailed descriptions of } \\
\text { matching methods }\end{array}$ & & $P=0.3054$ \\
\hline \multirow[t]{2}{*}{ Zhang ZX [36] } & 2012 & China & East Asian & Case: patients diagnosed with Rome III criteria & AS-PCR & \\
\hline & & & & Control: healthy volunteers matched for age, race and gender & & $P=0.4531$ \\
\hline \multirow[t]{2}{*}{ Kumar S [37] } & 2012 & India & Indian & Case: patients diagnosed with Rome III criteria & AS-PCR & \\
\hline & & & & $\begin{array}{l}\text { Control: healthy controls without detailed descriptions of } \\
\text { matching methods }\end{array}$ & & $P=0.3093$ \\
\hline \multirow[t]{2}{*}{ Saito YA [38] } & 2012 & USA & Mostly Caucasia & Case: patients diagnosed with Rome III criteria & AS-PCR & \\
\hline & & & & Control: healthy examinees matched for age, race and gender & & $P=0.5924$ \\
\hline \multirow[t]{2}{*}{ Colucci R [39] } & 2013 & Italy & Caucasian & Case: patients diagnosed with Rome III criteria & AS-PCR & \\
\hline & & & & $\begin{array}{l}\text { Control: healthy controls without detailed descriptions of } \\
\text { matching methods }\end{array}$ & & $P=0.6459$ \\
\hline \multirow[t]{2}{*}{ Shiotani A [40] } & 2013 & Japan & East Asian & Case: patients diagnosed with Rome III criteria & AS-PCR & \\
\hline & & & & $\begin{array}{l}\text { Control: healthy controls without detailed descriptions of } \\
\text { matching methods }\end{array}$ & & $P=0.5780$ \\
\hline \multirow[t]{2}{*}{ Farjadian S [41] } & 2013 & Iran & Iranian & Case: patients diagnosed with Rome III criteria & AS-PCR & \\
\hline & & & & Control: healthy examinees matched for age, race and gender & & $P=0.2284$ \\
\hline
\end{tabular}

SNP: single nucleotide polymorphism; PCR-RFLP: polymerase chain reaction-restriction fragment length polymorphism; AS-PCR: allele-specific polymerase chain reaction; HWE test: Hardy-Weinberg equilibrium test; NC: not able to calculated from raw data.

study conducted in the Mexican population did not evaluate the effect of the 5-HTTLPR polymorphism on IBS-C subtype.

\section{Meta-analysis about IBS-D and IBS-M}

No significant association was found between this polymorphism and IBS-D and IBS-M in the overall population. In subgroup analysis, there was no significant association between this polymorphism and IBS-C and IBS-M development in the Caucasian, East Asian, Indian and Iranian population. In the analysis between the 5HTTLPR polymorphism and IBS-D in the Turkish population, only the LS vs SS contrast showed a positive result $(\mathrm{OR}=8.471,95 \%=1.811-39.626, P=0.007$, Bon $=0.042)$. No association was found between this polymorphism and IBS-M development in the Turkish population. The study conducted in the Mexican population did not evaluate the effect of this polymorphism on IBS-D and IBS-M subtypes.

\section{Sensitivity analysis}

After excluding studies not fulfilling HWE or not providing HWE data, the conclusions of our meta-analysis were not changed. The conclusions were not changed either when we used both a fixed and a random model to perform meta-analyses.

\section{Evaluation of publication bias}

The Begg funnel plot was symmetry in the overall IBS analysis, as shown in Figure 4. Begger's test showed no publication bias in the overall IBS analysis $(P=0.529)$.

\section{Discussion}

Gut motility dysfunction, visceral hypersensitivity and psychopathological factors have been implicated to play key roles in the development of IBS [1,3]. Recently, some scholars integrated the above factors and postulated a model called brain-gut axis (BGA), which is bi-directional and constitutes the enteric nervous system (ENS) and the gut wall, CNS and the hypothalamus-pituitary-adrenal (HPA) axis [42]. BGA dysfunction at each level is believed to be involved in the pathophysiology of IBS [43]. Psychopathological factors cause gut motility dysfunction, abnormal mucosa secretion and visceral hypersensitivity via BGA and vice versa. So BGA model may be the optimal model in understanding IBS pathophysiology and can explain most of the presentations of IBS frequently accompanied with psychological conditions.

Neural, endocrine and neuro-immune pathways are all involved in BGA. And neurotransmitters are involved in all the three pathways, so inappropriate neurotransmitter secretion could cause BGA dysfunction and plays a key role in IBS development. Serotonin existing in not only GI tracts but also CNS is one of these neurotransmitters. 
Table 2 Meta-analyses of the association between the 5-HTTLPR polymorphism and the presence of IBS

\begin{tabular}{|c|c|c|c|c|c|c|c|c|c|c|c|}
\hline & \multirow[t]{2}{*}{ Ethnicity } & \multirow[t]{2}{*}{ Cases/Controls } & \multicolumn{3}{|l|}{ L vs $S$} & \multicolumn{3}{|l|}{ LL vs SS } & \multicolumn{3}{|l|}{ LL vs LS } \\
\hline & & & $(\mathrm{OR}, 95 \% \mathrm{Cl})$ & $P$ (Model) & Bon & $(\mathrm{OR}, 95 \% \mathrm{Cl})$ & $P$ (Model) & Bon & $(\mathrm{OR}, 95 \% \mathrm{Cl})$ & $P$ (Model) & Bon \\
\hline \multicolumn{12}{|l|}{ IBS } \\
\hline 25 studies & Overall & $3443 / 3359$ & $(0.993,0.867-1.138)$ & $P=0.923(\mathrm{R})$ & 1.000 & $(1.009,0.772-1.319)$ & $P=0.948(\mathrm{R})$ & 1.000 & $(1.284,1.040-1.585)$ & $P=0.020(\mathrm{R})$ & 0.120 \\
\hline 11 studies & $\mathrm{Cau} / \mathrm{M} \mathrm{Cau}$ & $1855 / 1590$ & $(1.008,0.859-1.183)$ & $P=0.919(\mathrm{R})$ & 1.000 & $(1.018,0.752-1.378)$ & $P=0.907(\mathrm{R})$ & 1.000 & $(1.288,1.079-1.536)$ & $P=0.005(\mathrm{~F})$ & 0.030 \\
\hline 9 studies & East Asian & 1138/1102 & $(1.151,0.901-1.471)$ & $P=0.261(\mathrm{R})$ & 1.000 & $(1.371,0.764-2.461)$ & $P=0.291(\mathrm{R})$ & 1.000 & $(1.528,0.849-2.751)$ & $P=0.157(\mathrm{R})$ & 0.942 \\
\hline 1 study & Turkish & $54 / 92$ & $(0.934,0.577-1.514)$ & $P=0.782$ & 1.000 & $(0.727,0.271-1.952)$ & $P=0.527$ & 1.000 & $(0.442,0.171-1.143)$ & $P=0.092$ & 0.552 \\
\hline 2 studies & Indian & $301 / 352$ & $(0.687,0.375-1.259)$ & $P=0.225(\mathrm{R})$ & 1.000 & $(0.588,0.241-1.435)$ & $P=0.243(\mathrm{R})$ & 1.000 & $(1.077,0.669-1.733)$ & $P=0.761(F)$ & 1.000 \\
\hline 1 study & Mexian & $45 / 123$ & $(0.482,0.256-0.910)$ & $P=0.024$ & 0.144 & $(0.290,0.082-1.030)$ & $P=0.056$ & 0.336 & $(0.313,0.073-1.337)$ & $P=0.117$ & 0.702 \\
\hline 1 study & Iranian & $50 / 100$ & $(1.083,0.670-1.751)$ & $P=0.744$ & 1.000 & $(1.168,0.466-2.931)$ & $P=0.740$ & 1.000 & $(2.246,0.976-5.165)$ & $P=0.057$ & 1.000 \\
\hline \multicolumn{12}{|l|}{ IBS-C } \\
\hline 20 studies & Overall & $992 / 2437$ & $(1.232,1.048-1.449)$ & $P=0.011(\mathrm{R})$ & 0.066 & $(1.570,1.147-2.148)$ & $P=0.005(\mathrm{~F})$ & 0.030 & $(1.658,1.180-2.331)$ & $P=0.004(\mathrm{R})$ & 0.024 \\
\hline 8 studies & Cau/M Cau & $462 / 841$ & $(1.270,1.060-1.520)$ & $P=0.009(\mathrm{~F})$ & 0.054 & $(1.627,1.109-2.388)$ & $P=0.013(\mathrm{~F})$ & 0.078 & $(1.269,0.956-1.685)$ & $P=0.100(F)$ & 1.000 \\
\hline 8 studies & East Asian & $393 / 1052$ & $(1.487,1.139-1.941)$ & $P=0.003(\mathrm{R})$ & 0.018 & $(2.575,1.741-3.808)$ & $P=0.000(\mathrm{~F})$ & 0.000 & $(3.084,2.017-4.715)$ & $P=0.000(\mathrm{~F})$ & 0.000 \\
\hline 1 study & Turkish & 26/92 & $(0.719,0.379-1.367)$ & $P=0.315$ & 1.000 & $(0.545,0.156-1.903)$ & $P=0.342$ & 1.000 & $(0.618,0.172-2.218)$ & $P=0.461$ & 1.000 \\
\hline 2 studies & Indian & $96 / 352$ & $(0.798,0.438-1.452)$ & $P=0.460(\mathrm{R})$ & 1.000 & $(0.676,0.330-1.384)$ & $P=0.284(F)$ & 1.000 & $(0.769,0.389-1.522)$ & $P=0.451(F)$ & 1.000 \\
\hline 1 study & Iranian & $15 / 100$ & $(0.796,0.367-1.725)$ & $P=0.563$ & 1.000 & $(0.730,0.181-2.951)$ & $P=0.659$ & 1.000 & $(2.133,0.522-8.714)$ & $P=0.291$ & 1.000 \\
\hline \multicolumn{12}{|l|}{ IBS-D } \\
\hline 21 studies & Overall & $1454 / 2813$ & $(0.932,0.771-1.126)$ & $P=0.466(\mathrm{R})$ & 1.000 & $(0.888,0.666-1.183)$ & $P=0.416(\mathrm{R})$ & 1.000 & $(1.209,0.973-1.502)$ & $P=0.086(\mathrm{R})$ & 0.516 \\
\hline 9 studies & Cau/M Cau & $806 / 1270$ & $(0.986,0.863-1.126)$ & $P=0.835(F)$ & 1.000 & $(0.921,0.706-1.200)$ & $P=0.540(\mathrm{~F})$ & 1.000 & $(1.251,1.005-1.556)$ & $P=0.045(\mathrm{~F})$ & 0.270 \\
\hline 8 studies & East Asian & $434 / 999$ & $(0.846,0.694-1.032)$ & $P=0.099(F)$ & 0.594 & $(0.785,0.498-1.239)$ & $P=0.299(F)$ & 1.000 & $(0.930,0.579-1.494)$ & $P=0.764(F)$ & 1.000 \\
\hline 1 study & Turkish & 18/92 & $(1.087,0.529-2.232)$ & $P=0.820$ & 1.000 & $(0.324,0.015-7.070)$ & $P=0.474$ & 1.000 & $(0.046,0.003-0.814)$ & $P=0.036$ & 0.216 \\
\hline 2 studies & Indian & $171 / 352$ & $(0.478,0.084-2.707)$ & $P=0.404(\mathrm{R})$ & 0.828 & $(0.494,0.069-3.514)$ & $P=0.481(R)$ & 1.000 & $(1.355,0.715-2.566)$ & $P=0.351(F)$ & 1.000 \\
\hline 1 study & Iranian & $25 / 100$ & $(1.325,0.710-2.471)$ & $P=0.377$ & 1.000 & $(1.565,0.504-4.856)$ & $P=0.439$ & 1.000 & $(3.333,1.159-9.586)$ & $P=0.025$ & 0.150 \\
\hline \multicolumn{12}{|l|}{ IBS-M } \\
\hline 17 studies & Overall & $486 / 2042$ & $(1.087,0.911-1.296)$ & $P=0.354(\mathrm{R})$ & 1.000 & $(1.170,0.830-1.651)$ & $P=0.370(\mathrm{R})$ & 1.000 & $(1.313,0.991-1.740)$ & $P=0.058(F)$ & 0.348 \\
\hline 6 studies & Cau/M Cau & $265 / 549$ & $(1.096,0.754-1.593)$ & $P=0.630(\mathrm{R})$ & 1.000 & $(1.153,0.538-2.471)$ & $P=0.714(\mathrm{R})$ & 1.000 & $(1.533,1.069-2.198)$ & $P=0.020(\mathrm{~F})$ & 0.120 \\
\hline 7 studies & East Asian & $167 / 949$ & $(1.030,0.785-1.351)$ & $P=0.830(F)$ & 1.000 & $(1.135,0.637-2.022)$ & $P=0.667(F)$ & 1.000 & $(0.906,0.507-1.617)$ & $P=0.737(F)$ & 1.000 \\
\hline 1 study & Turkish & $10 / 92$ & $(2.038,0.795-5.225)$ & $P=0.138$ & 0.828 & $(4.909,0.480-50.178)$ & $P=0.180$ & 1.000 & $(0.773,0.175-3.415$ & $P=0.734$ & 1.000 \\
\hline 2 studies & Indian & $34 / 352$ & $(1.022,0.616-1.697)$ & $P=0.932(F)$ & 1.000 & $(1.157,0.452-2.965)$ & $P=0.761(F)$ & 1.000 & $(1.684,0.662-4.280$ & $P=0.274(F)$ & 1.000 \\
\hline 1 study & Iranian & $10 / 100$ & $(1.041,0.415-2.610)$ & $P=0.932$ & 1.000 & $(1.095,0.141-8.485)$ & $P=0.931$ & 1.000 & $(0.889,0.166-4.755$ & $P=0.891$ & 1.000 \\
\hline
\end{tabular}


Table 3 Meta-analyses of the association between the 5-HTTLPR polymorphism and the presence of IBS

\begin{tabular}{|c|c|c|c|c|c|c|c|c|c|c|c|}
\hline & \multirow[t]{2}{*}{ Ethnicity } & \multirow[t]{2}{*}{ Cases/Controls } & \multicolumn{3}{|l|}{ LS vs SS } & \multicolumn{3}{|l|}{ LL vs LS/SS } & \multicolumn{3}{|l|}{ LL/LS vs SS } \\
\hline & & & $(\mathrm{OR}, 95 \% \mathrm{Cl})$ & $P$ (Model) & Bon & $(\mathrm{OR}, 95 \% \mathrm{Cl})$ & $P$ (Model) & Bon & $(\mathrm{OR}, 95 \% \mathrm{Cl})$ & $P$ (Model) & Bon \\
\hline \multicolumn{12}{|l|}{ IBS } \\
\hline 25 studies & Overall & $3443 / 3359$ & $(0.814,0.681-0.973)$ & $P=0.023(\mathrm{R})$ & 0.138 & $(1.141,0.935-1.391)$ & $P=0.195(\mathrm{R})$ & 1.000 & $(0.883,0.739-1.055)$ & $P=0.171(\mathrm{R})$ & 1.000 \\
\hline 11 studies & Cau/M Cau & $1855 / 1590$ & $(0.796,0.566-1.119)$ & $P=0.189(\mathrm{R})$ & 1.000 & $(1.506,1.112-2.039)$ & $P=0.070(F)$ & 0.420 & $(0.874,0.643-1.189)$ & $P=0.391(\mathrm{R})$ & 1.000 \\
\hline 9 studies & East Asian & 1138/1102 & $(0.892,0.727-1.094)$ & $P=0.272(\mathrm{R})$ & 1.000 & $(1.456,0.831-2.549)$ & $P=0.189(\mathrm{R})$ & 1.000 & $(0.999,0.828-1.207)$ & $P=0.995(F)$ & 1.000 \\
\hline 1 study & Turkish & $54 / 92$ & $(1.647,0.774-3.505)$ & $P=0.195$ & 1.000 & $(0.553,0.227-1.348)$ & $P=0.193$ & 1.000 & $(1.286,0.636-2.599)$ & $P=0.484$ & 1.000 \\
\hline 2 studies & Indian & $301 / 352$ & $(0.542,0.284-1.034)$ & $P=0.063(\mathrm{R})$ & 0.378 & $(0.763,0.494-1.178)$ & $P=0.223(F)$ & 1.000 & $(0.555,0.272-1.130)$ & $P=0.105(\mathrm{R})$ & 1.000 \\
\hline 1 study & Mexian & $45 / 123$ & $(0.929,0.373-2.316)$ & $P=0.875$ & 1.000 & $(0.295,0.084-.032)$ & $P=0.056$ & 0.336 & $(0.581,0.268-1.259)$ & $P=0.169$ & 1.000 \\
\hline 1 study & Iranian & $50 / 100$ & $(0.520,0.226-1.197)$ & $P=0.124$ & 0.744 & $(1.770,0.824-3.803)$ & $P=0.143$ & 0.858 & $(0.697,0.325-1.495)$ & $P=0.354$ & 1.000 \\
\hline \multicolumn{12}{|l|}{ IBS-C } \\
\hline 20 studies & Overall & $992 / 2437$ & $(0.926,0.708-1.212)$ & $P=0.578(\mathrm{R})$ & 1.000 & $(1.545,1.187-2.012)$ & $P=0.001(\mathrm{R})$ & 0.006 & $(1.119,0.890-1.406)$ & $P=0.335(\mathrm{R})$ & 1.000 \\
\hline 8 studies & Cau/M Cau & $462 / 841$ & $(1.266,0.883-1.815)$ & $P=0.199(F)$ & 1.000 & $(1.327,1.027-1.715)$ & $P=0.031(\mathrm{~F})$ & 0.186 & $(1.398,0.994-1.968)$ & $P=0.054(F)$ & 0.324 \\
\hline 8 studies & East Asian & $393 / 1052$ & $(0.906,0.672-1.220)$ & $P=0.514(F)$ & 1.000 & $(2.759,1.933-3.938)$ & $P=0.000(\mathrm{~F})$ & 0.000 & $(1.250,0.964-1.622)$ & $P=0.093(F)$ & 0.558 \\
\hline 1 study & Turkish & $26 / 92$ & $(0.882,0.337-2.307)$ & $P=0.799$ & 1.000 & $(0.579,0.180-1.860)$ & $P=0.358$ & 1.000 & $(0.750,0.312-1.803)$ & $P=0.165$ & 0.990 \\
\hline 2 studies & Indian & $96 / 352$ & $(0.783,0.185-3.324)$ & $P=0.740(\mathrm{R})$ & 1.000 & $(0.726,0.378-1.394)$ & $P=0.336(F)$ & 1.000 & $(0.753,0.213-2.661)$ & $P=0.660(\mathrm{R})$ & 1.000 \\
\hline 1 study & Iranian & $15 / 100$ & $(0.342,0.095-1.234)$ & $P=0.101$ & 0.606 & $(1.368,0.395-4.734)$ & $P=0.621$ & 1.000 & $(0.448,0.144-1.391)$ & $P=0.165$ & 0.990 \\
\hline \multicolumn{12}{|l|}{ IBS-D } \\
\hline 21 studies & Overall & $1454 / 2813$ & $(0.863,0.619-1.205)$ & $P=0.387(\mathrm{R})$ & 1.000 & $(1.042,0.862-1.260)$ & $P=0.668(\mathrm{R})$ & 1.000 & $(0.885,0.648-1.209)$ & $P=0.443(\mathrm{R})$ & 1.000 \\
\hline 9 studies & Cau/M Cau & $806 / 1270$ & $(0.854,0.548-1.332)$ & $P=0.423(\mathrm{R})$ & 1.000 & $(1.113,0.914-1.357)$ & $P=0.286(F)$ & 1.000 & $(0.922,0.626-1.357)$ & $P=0.680(\mathrm{R})$ & 1.000 \\
\hline 8 studies & East Asian & 434/999 & $(0.896,0.686-1.171)$ & $P=0.423(F)$ & 1.000 & $(0.850,0.561-1.289)$ & $P=0.444(F)$ & 1.000 & $(0.843,0.656-1.083)$ & $P=0.181(F)$ & 1.000 \\
\hline 1 study & Turkish & $18 / 92$ & $(8.471,1.811-39.626)$ & $P=0.007$ & 0.042 & $(0.085,0.005-1.462)$ & $P=0.089$ & 0.534 & $(5.143,1.115-23.714)$ & $P=0.036$ & 0.096 \\
\hline 2 studies & Indian & $171 / 352$ & $(0.321,0.024-4.370)$ & $P=0.394(\mathrm{R})$ & 1.000 & $(0.680,0.214-2.159)$ & $P=0.513(F)$ & 1.000 & $(0.371,0.036-3.819)$ & $P=0.405(\mathrm{R})$ & 1.000 \\
\hline 1 study & Iranian & $25 / 100$ & $(0.469,0.152-1.445)$ & $P=0.187$ & 1.000 & $(2.508,0.986-6.380)$ & $P=0.054$ & 0.324 & $(0.768,0.286-2.066)$ & $P=0.601$ & 1.000 \\
\hline \multicolumn{12}{|l|}{ IBS-M } \\
\hline 17 studies & Overall & $486 / 2042$ & $(0.920,0.706-1.199)$ & $P=0.538(F)$ & 1.000 & $(1.186,0.887-1.584)$ & $P=0.250(\mathrm{R})$ & 1.000 & $(0.971,0.760-1.242)$ & $P=0.817(F)$ & 1.000 \\
\hline 6 studies & Cau/M Cau & $265 / 549$ & $(0.728,0.476-1.112)$ & $P=0.142(F)$ & 0.852 & $(1.172,0.707-1.942)$ & $P=0.539(\mathrm{R})$ & 1.000 & $(0.834,0.563-1.237)$ & $P=0.368(F)$ & 1.000 \\
\hline 7 studies & East Asian & $167 / 949$ & $(1.106,0.750-1.632)$ & $P=0.611(F)$ & 1.000 & $(1.000,0.590-1.696)$ & $P=1.000(F)$ & 1.000 & $(1.082,0.754-1.554)$ & $P=0.669(F)$ & 1.000 \\
\hline 1 study & Turkish & $10 / 92$ & $(6.353,0.727-55.545)$ & $P=0.095$ & 0.570 & $(1.364,0.325-5.726)$ & $P=0.672$ & 1.000 & $(5.786,0.703-47.626)$ & $P=0.103$ & 0.618 \\
\hline 2 studies & Indian & $34 / 352$ & $(0.695,0.309-1.562)$ & $P=0.379(F)$ & 1.000 & $(1.421,0.611-3.303)$ & $P=0.415(F)$ & 1.000 & $(0.817,0.393-1.698)$ & $P=0.588$ & 1.000 \\
\hline 1 study & Iranian & $10 / 100$ & $(1.232,0.231-6.560)$ & $P=0.807$ & 1.000 & $(0.940,0.186-4.764)$ & $P=0.941$ & 1.000 & $(1.195,0.237-6.025)$ & $P=0.829$ & 1.000 \\
\hline
\end{tabular}




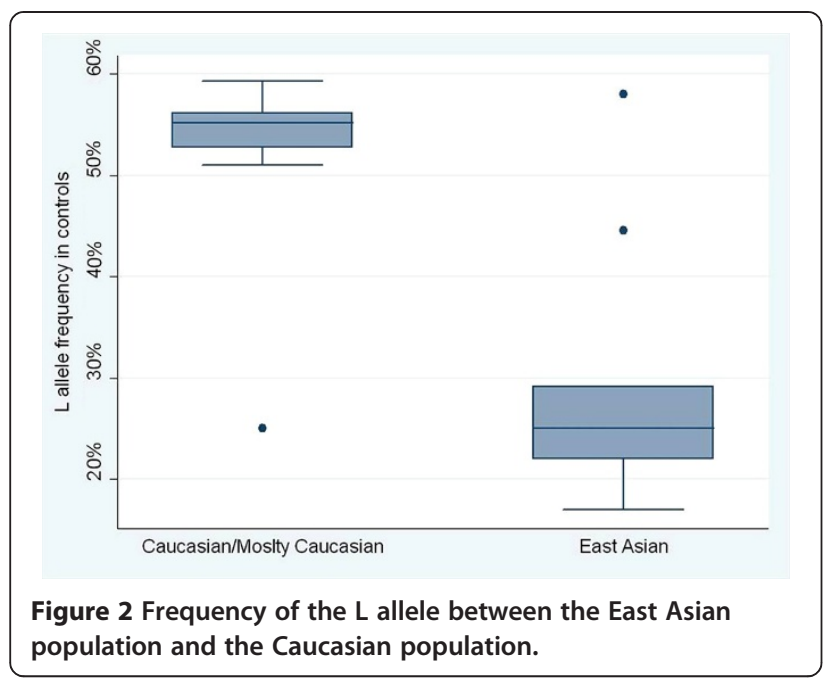

Study

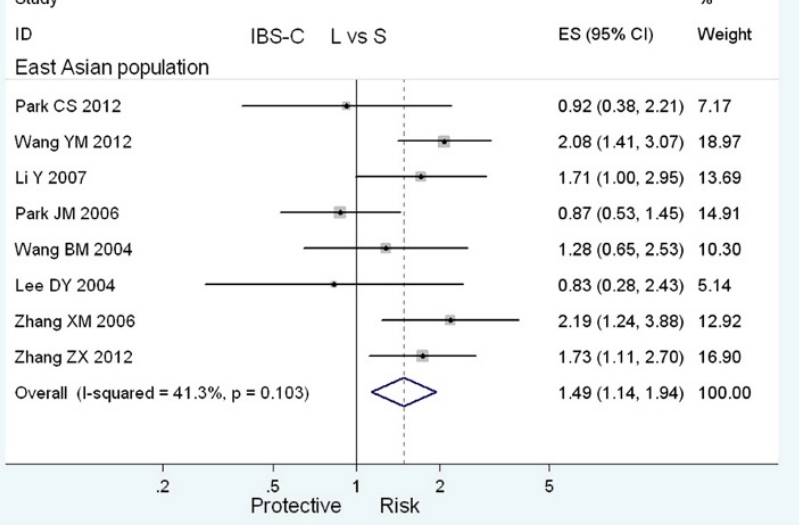

sudy

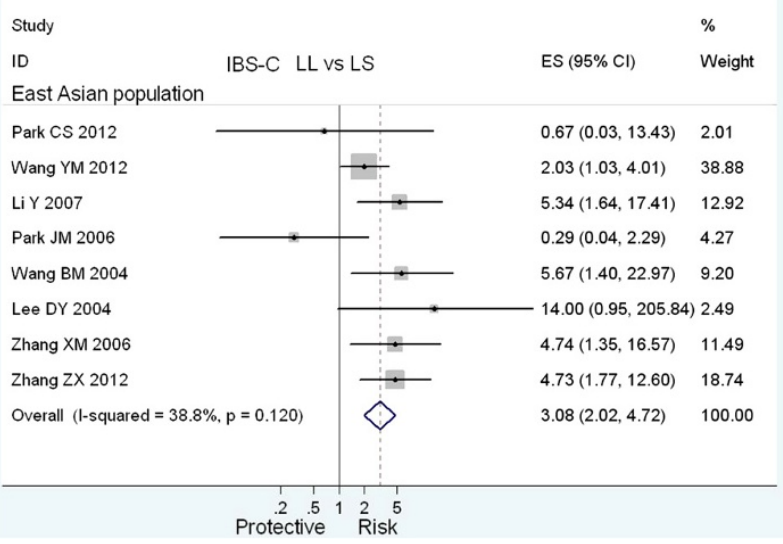

Figure 3 Meta-analyses with positive results of the association between the 5-HTTLPR polymorphism and the presence of IBS-C in the East Asian population.
Changes of the serotonin levels in GI tracts can affect visceral sensitivity and gut motility $[7,44]$. Previous studies have also showed that elevated plasma serotonin is associated with IBS-D and decreased plasma serotonin is associated with IBS-C [7]. Once serotonin is secreted from EC cells, SERT will be activated to reuptake serotonin back into EC cells and attenuate the effect of serotonin in GI tracts subsequently [8]. The L variation of 5 -HTTLPR is found to increase the expression of SERT gene and enhance SERT activity consequently [9-11,34]. The enhanced SERT activity would take up serotonin and weaken its effects in promoting gut secretion and motility. So compared with S allele and SS genotype carriers, L allele and LL genotype carriers are likely to be more susceptible to IBS-C. The results of our metaanalysis support this hypothesis. However, the effect of 5-HTTLPR on IBS-C is population dependent and the positive association is only present in the East Asian population but not in the Caucasian population. We also found that $\mathrm{L}$ allele frequency of the East Asian population controls was significantly lower than that of the
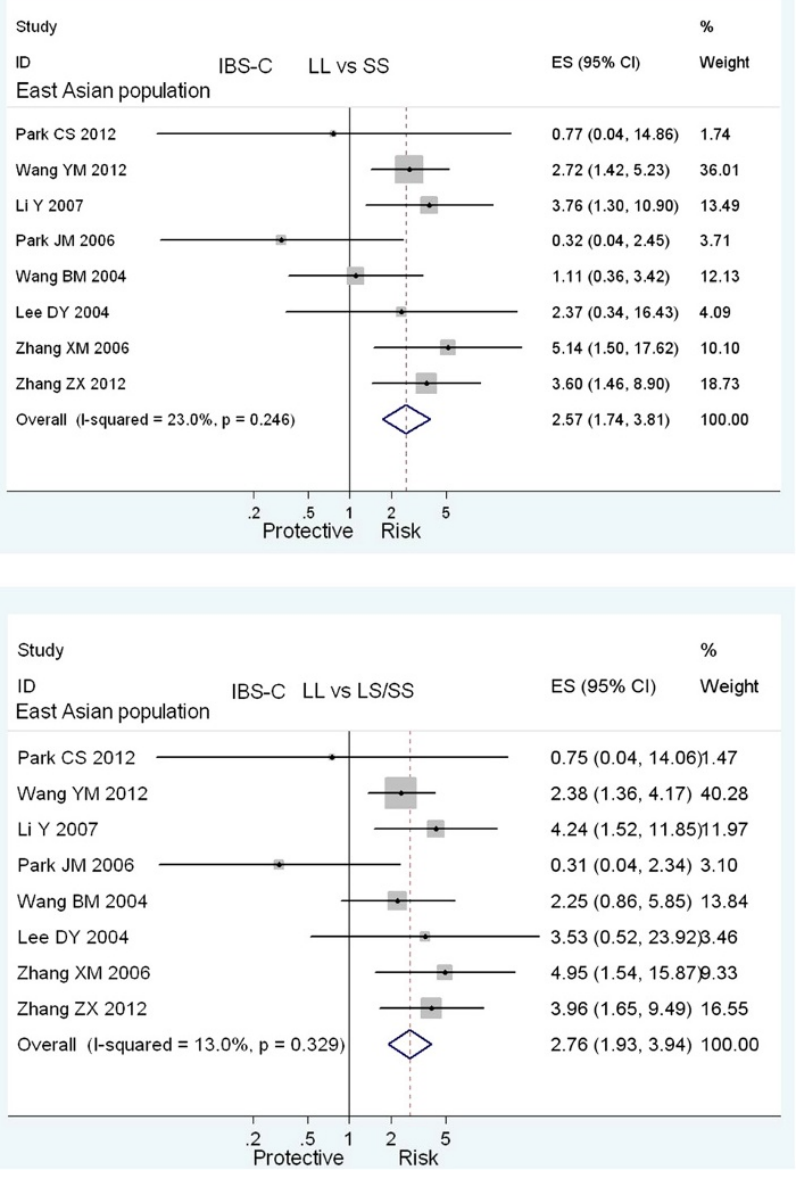

East Asian population IBS-C LL vs LS/SS ES $(95 \% \mathrm{Cl}) \quad$ Weight 


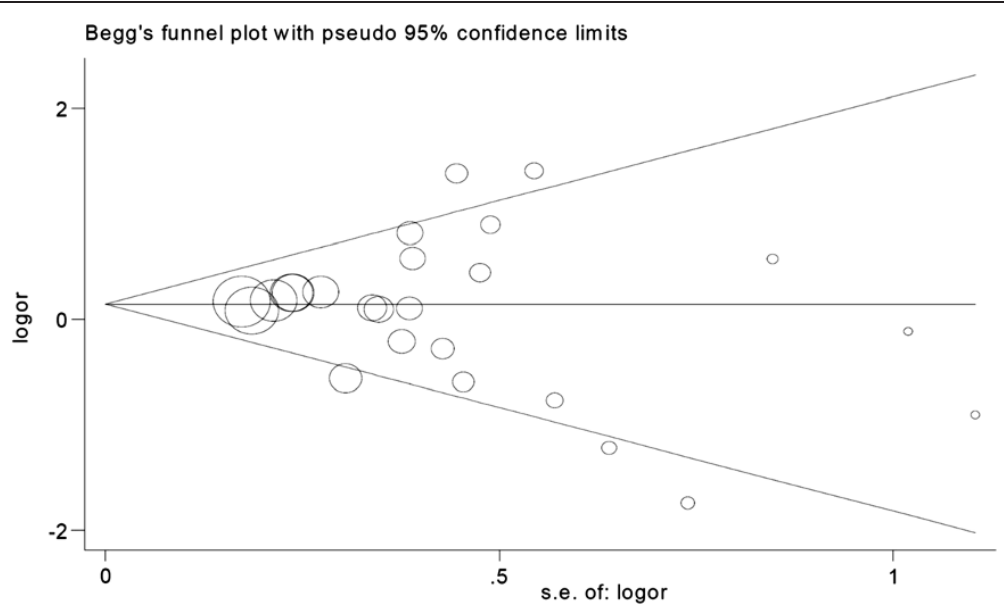

Figure 4 Begg funnel plot of the overall IBS analysis LL vs LS/SS.

Caucasian population controls. So the different allelic frequency between the two populations may account for this phenomenon. Furthermore, there is a higher prevalence of psychological and psychiatric disorders among IBS patients [45]. The state of CNS can also affect the development of IBS. However, the effect of the 5-HTTLPR variant on CNS is different from that on GI tracts in IBS development. A meta-analysis demonstrated that the $\mathrm{S}$ variant was associated with heightened amygdala activation which would predispose $\mathrm{S}$ carriers to stress-related psychiatric disorders [46]. Another meta-analysis also showed a positive association between the $\mathrm{S}$ allele and an increased risk of developing depression under stress [47]. The $\mathrm{S}$ allele may increase the risk of IBS-C development through the CNS pathway. So the opposite effect of 5HTTLPR variant on GI tracts and CNS during IBS-C development may also account for some variations among studies assessing the association between the 5-HTTLPR variant and IBS-C. Future studies should exclude the psychological confounding factors or stratify analyses based on psychological conditions. IBS may be further categorized into subtypes based on psychological conditions besides of the Rome criteria. Additionally, an adenine to guanine polymorphism in the $\mathrm{L}$ allele has been recently identified and only the adenine $\mathrm{L}$ allele but not the guanine $\mathrm{L}$ allele is found to increase the activity of SERT [48]. This internal $\mathrm{L}$ allele polymorphism may attenuate the effect of the L allele on IBS-C development. Only one small sample size study included in our meta-analysis evaluated the effect of internal L allele polymorphism on IBS development and showed a negative result. Future studies should further assess the effect of the 5-HTTLPR polymorphism on IBS-C according to the internal $\mathrm{L}$ allele polymorphism. Moreover, a study indicates that the $\mathrm{S}$ allele is associated with higher pain sensory ratings during rectal distension studies in healthy controls and IBS patients, and the increased sensation ratings in carriers of the $S$ allele are not caused by lower rectal compliance [27]. Different genotype and allele may have different effects on gut motility and intestinal sensation respectively. This phenomenon also indicates the complexity of IBS and the necessity to classifying IBS based on pathophysiology.

Clarifying genotypes of the 5-HTTLPR polymorphism also has clinical implications. One study found that IBS-C patients with the LL genotype responded poorly to treatment with the 5-HT4 receptor agonist, tegaserod [25]. Another study investigating rectal smooth muscle contractions found that IBS patients with the SS genotype showed more increase in phasic contractions compared with patients with the LL genotype after administration of the 5-HT4 receptor agonist, mosapride [49]. Determining the genotype of the 5-HTTLPR polymorphism may be of value to the prognosis and the prediction of treatment response in a IBS-C patient.

The conclusions of this meta-analysis are a little different from the previous meta-analyses. As we know, this meta-analysis is the latest one and has the largest sample size. So the result of our meta-analysis is more likely convincing. However, the conclusions of this meta-analysis should be interpreted cautiously due to some limitations. Firstly, selection bias could not be excluded as only seven studies employed measures to exclude IBS in the controls. Secondly, only four studies used the DNA sequencing method to confirm genotypes, and two studies used blindness measures. Information bias is inevitable. Thirdly, most of the included studies did not describe the age and gender distribution between cases and controls. Confounding factors could not be excluded. Moreover, heterogeneity existed in some contrasts which might also affect the validity of this meta-analysis. Although the Egger's test showed no publication bias existing, the data of two abstracts were 
not included in this meta-analysis for failing to provide genotype frequencies. Thus a reporting bias existed. Moreover, we employed the Bonferroni adjustments method to avoid false positive results, the risk of false negative results occurrence would be increased. So when interpreting the negative results of our metaanalysis, we should be cautious.

\section{Conclusions}

In summary, in despite of the above limitations, this metaanalysis shows a positive association between the $\mathrm{L}$ allele and LL genotype of 5-HTTLPR mutation and IBS-C in the East Asian population but not in the Caucasian population. These results offer some insights into gene functions affecting IBS susceptibility and some clues in IBS management, especially in the East Asian population.

\section{Additional files}

Additional file 1: The PRISMA Statement.

Additional file 2: The searching process of Pubmed, Embase and Web of Science.

\section{Competing interests}

The authors declare that they have no competing interests.

\section{Authors' contributions}

Conceived and designed the experiments: ZFZ and ZJD. Performed the experiments: ZFZ, LXW, DY, GZ, LZ. Searched, selected, analyzed the data: ZFZ, LXW, DY, GZ, LZ. Wrote the paper: ZFZ, LXW, DY. All authors read and approved the final manuscript.

\section{Acknowledgment}

We should acknowledge librarian Jiao He of the first affiliated hospital of Dalian Medical University for the retrieving of published articles.

\section{Author details}

${ }^{1}$ Department of Gastroenterology, The First Affiliated Hospital of Dalian Medical University, 116000 Dalian, Liaoning province, China. ${ }^{2}$ Department of Respiratory Diseases, The First Affiliated Hospital of Dalian Medical University, 116000 Dalian, Liaoning province, China.

Received: 24 October 2013 Accepted: 30 January 2014 Published: 10 February 2014

\section{References}

1. Drossman DA, Camilleri M, Mayer EA, et al: AGA technical review on irritable bowel syndrome. Gastroenterology 2002, 123:2108-2131.

2. Guilera M, Balboa A, Mearin F: Bowel habit subtypes and temporal patterns in irritable bowel syndrome: systematic review. Am J Gastroenterol 2005, 100:1174-1184.

3. Longstreth GF, Thompson WG, Chey WD, et al: Functional bowel disorders. Gastroenterology 2006, 130:1480-1491.

4. Wilson $S$, Roberts $L$, Roalfe $A$, et al: Prevalence of irritable bowel syndrome: a community survey. Br J Gen Pract 2004, 54:495-502.

5. Robin S: Clinical update: Irritable bowel syndrome. Lancet 2007, 369:1586-1588.

6. Kim DY, Camilleri M: Serotonin: a mediator of the brain-gut connection. Am J Gastroenterol 2000, 95:2698-2709.

7. Atkinson W, Lockhart S, Whorwell PJ, et al: Altered 5-hydroxytryptamine signaling in patients with constipation- and diarrhea-predominant irritable bowel syndrome. Gastroenterology 2006, 130:34-43.

8. Bertrand PP, Bertrand RL: Serotonin release and uptake in the gastrointestinal tract. Auton Neurosci 2010, 153:47-57.
9. Heils A, Teufel A, Petri $S$, et al: Allelic variation of human serotonin transporter gene expression. J Neurochem 1996, 66:2621-2624.

10. Yeo A, Boyd P, Lumsden S, et al: Association between a functional polymorphism in the serotonin transporter gene and diarrhoea predominant irritable bowel syndrome in women. Gut 2004, 53:1452-1458.

11. Park JM, Choi MG, Park JA, et al: Serotonin transporter gene polymorphism and irritable bowel syndrome. Neurogastroenterol Motil 2006, 18:995-1000.

12. Kim HJ, Camilleri M, Carlson PJ, et al: Association of distinct alpha(2) adrenoceptor and serotonin transporter polymorphisms with constipation and somatic symptoms in functional gastrointestinal disorders. Gut 2004, 53:829-37.

13. Van Kerkhoven LA, Laheij RJ, Jansen JB: Meta-analysis: a functional polymorphism in the gene encoding for activity of the serotonin transporter protein is not associated with the irritable bowel syndrome. Aliment Pharmacol Ther 2007, 26:979-986.

14. Dai C, Zheng CQ, Jiang M: Letter: serotonin transporter gene polymorphisms and the irritable bowel syndrome. Aliment Pharmacol Ther 2013, 37(6):657-658.

15. Areeshi MY, Haque S, Panda AK, Mandal RK: A serotonin transporter gene (SLC6A4) polymorphism is associated with reduced risk of irritable bowel syndrome in American and Asian population: a meta-analysis. PLoS One 2013, 8(9):e75567.

16. Higgins JPT, Green S (Eds): Cochrane Handbook for Systematic Reviews of Interventions Version 5.1.0 [updated March 2011]. The Cochrane Collaboration. Accessed 2011 June 30 http://www.cochrane.org/training/ cochrane-handbook

17. Egger M, Smith GD, Schneider M, et al: Bias in meta-analysis detected by a simple, graphical test. Br Med J 1997, 315:629-634

18. Sterne JA, Egger M: Funnel plots for detecting bias in meta-analysis: guidelines on choice of axis. J Clin Epidemiol 2001, 54:1046-1055.

19. Holm S: A simple sequentially rejective bonferroni test procedure. Scand J Stat 1979, 6:65-70.

20. Pata C, Erdal ME, Derici E, et al: Serotonin transporter gene polymorphism in irritable bowel syndrome. Am J Gastroenterol 2002, 97(7):1780-1784.

21. Lee DY, Park H, Kim WH, et al: Serotonin transporter gene polymorphism in healthy adults and patients with irritable bowel syndrome. Korean J Gastroenterol 2004, 43(1):18-22.

22. Wang BM, Wang YM, Zhang WM, et al: Serotonin transporter gene polymorphism in irritable bowel syndrome. Zhonghua Nei Ke Za Zhi 2004, 43(6):439-441

23. Zhang XM, Lin ZH: Relationship between serotonin transporter gene polymorphism and irritable bowel syndrome. Shijie Huaren Xiaohua Zazhi 2006, 14(18):1790-1794.

24. Whitehead WE, Palsson OS, Thiwan SI, et al: Short-allele serotonin transporter gene polymorphism is associated with elevated serotonin and is less common in IBS with constipation compared to healthy controls. Gastroenterology 2006, 130(4):A513.

25. Li Y, Nie $Y$, Xie J, et al: The association of serotonin transporter genetic polymorphisms and irritable bowel syndrome and its influence on tegaserod treatment in Chinese patients. Dig Dis Sci 2007, 52(11):2942-2949.

26. Saito YA, Locke GR 3rd, Zimmerman JM, et al: A genetic association study of 5-HTT LPR and GNbeta3 C825T polymorphisms with irritable bowel syndrome. Neurogastroenterol Motil 2007, 19(6):465-470.

27. Camilleri M, Busciglio I, Carlson P, et al: Candidate genes and sensory functions in health and irritable bowel syndrome. Am J Physiol Gastrointest Liver Physiol 2008, 295(2):G219-225.

28. Kohen $\mathrm{R}$, Jarrett ME, Cain KC, et al: The serotonin transporter polymorphism rs 25531 is associated with irritable bowel syndrome. Dig Dis Sci 2009, 54(12):2663-2770.

29. Schmulson M, Farfan-Labone B, Charua-Guindic L, et al: The presence of the short allele in the promoter region of the SERT-P is associated with IBS among females in a Latin population. Neurogastroenterol Motil 2009, 21:S90.

30. Niesler B, Kapeller J, Fell C, et al: 5-HTTLPR and STin2 polymorphisms in the serotonin transporter gene and irritable bowel syndrome: effect of bowel habit and sex. Eur J Gastroenterol Hepatol 2010, 22(7):856-861.

31. Sikander A, Rana SV, Sinha SK, et al: Serotonin transporter promoter variant: Analysis in Indian IBS patients and control population. J Clin Gastroenterol 2009, 43(10):957-961.

32. Markoutsaki T, Karantanos T, Gazouli M, et al: 5-HT2A receptor gene polymorphisms and irritable bowel syndrome. J Clin Gastroenterol 2011, 45(6):514-517. 
33. Jiang ZD, Dupont AW, DuPont HL, et al: Genetic polymorphisms in serotonin transporter and G-protein genes in patients with irritable bowel syndrome. Gastroenterology 2012, 142(5):S562.

34. Wang YM, Chang Y, Chang YY, et al: Serotonin transporter gene promoter region polymorphisms and serotonin transporter expression in the colonic mucosa of irritable bowel syndrome patients. Neurogastroenterol Motil 2012, 24(6):560-565.

35. Park CS, Uhm JH: Polymorphisms of the serotonin transporter gene and G-protein $\beta 3$ subunit gene in korean children with irritable bowel syndrome and functional dyspepsia. Gut Liver 2012, 6(2):223-228.

36. Zhang ZX, Xie J, Huang CB, et al: Serotonin transporter polymorphism in constipation predominant irritable bowel syndrome and its influence on clinical effect of tegaserod. Shandong Yiyao 2012, 52(25):16-18.

37. Kumar S, Ranjan P, Mittal B, et al: Serotonin transporter gene (SLC6A4) polymorphism in patients with irritable bowel syndrome and healthy controls. J Gastrointestin Liver Dis 2012, 21(1):31-38

38. Saito YA, Larson JJ, Atkinson EJ, et al: The role of 5-HTT LPR and GNB3 $825 \mathrm{C}>\mathrm{T}$ polymorphisms and gene-environment interactions in irritable bowel syndrome (IBS). Dig Dis Sci 2012, 57(10):2650-2657.

39. Colucci R, Gambaccini D, Ghisu N, et al: Influence of the serotonin transporter $5 \mathrm{HTTLPR}$ polymorphism on symptom severity in irritable bowel syndrome. PLOS ONE 2013, 8(2):e54831.

40. Shiotani A, Kusunoki H, Kimura Y, et al: S100A Expression and interleukin10 polymorphisms Are associated with ulcerative colitis and diarrhea predominant irritable bowel syndrome. Dig Dis Sci 2013, 18 doi:10.1007/s10620-013-2677-y. [Epub ahead of print]

41. Farjadian S, Fakhraei B, Moeini M, et al: Serotonin transporter gene polymorphisms in Southwestern Iranian patients with irritable bowel syndrome. Arab J Gastroenterol 2013, 14(2):59-62.

42. Collins SM, Bercik P: The relationship between intestinal microbiota and the central nervous system in normal gastrointestinal function and disease. Gastroenterology 2009, 136(6):2003-2014.

43. Clarke G, Quigley EM, Cryan JF, et al: Irritable bowel syndrome: towards biomarker identification. Trends Mol Med 2009, 15(10):478-489.

44. Zemlan FP, Murphy AZ, Behbehani MM: 5-HT1A receptors mediate the effect of the bulbospinal serotonin system on spinal dorsal horn nociceptive neurones. Pharmacology 1994, 48:1-10.

45. Arebi N, Gurmany S, Bullas D, et al: Review article: the psychoneuroimmunology of irritable bowel syndrome-an exploration of interactions between psychological, neurological and immunological observations. Aliment Pharmacol Ther 2008, 28(7):830-840.

46. Munafò MR, Brown SM, Hariri AR: Serotonin transporter (5-HTTLPR) genotype and amygdala activation: a meta-analysis. Biol Psychiatry 2008, 63:852-857.

47. Karg K, Burmeister M, Shedden $K$, et al: The serotonin transporter promoter variant (5-HTTLPR), stress, and depression meta-analysis revisited: evidence of genetic moderation. Arch Gen Psychiatry 2011, 68:444-454.

48. Lipsky RH, Hu XZ, Goldman D: Additional functional variation at the SLC6 A4 gene. Am J Med Genet B Neuropsychiatr Genet 2009, 150:153.

49. Kanazawa M, Watanabe S, Tana C, Komuro H, Aoki M, Fukudo S: Effect of 5-HT4 receptor agonist mosapride citrate on rectosigmoid sensorimotor function in patients with irritable bowel syndrome. Neurogastroenterol Motil 2011, 23(8):754-e332.

\section{doi:10.1186/1471-230X-14-23}

Cite this article as: Zhang et al:: The serotonin transporter gene

polymorphism (5-HTTLPR) and irritable bowel syndrome: a

meta-analysis of 25 studies. BMC Gastroenterology 2014 14:23.

\section{Submit your next manuscript to BioMed Central and take full advantage of:}

- Convenient online submission

- Thorough peer review

- No space constraints or color figure charges

- Immediate publication on acceptance

- Inclusion in PubMed, CAS, Scopus and Google Scholar

- Research which is freely available for redistribution 